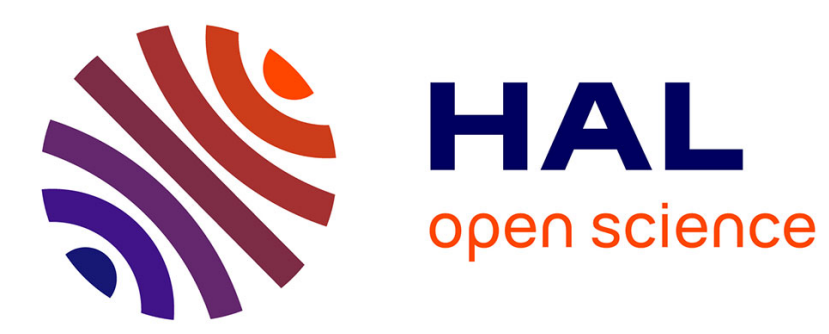

\title{
The Tricks of the Chameleon. Unexpected Symmetry of the Diffraction Pattern
}

\author{
Massimo Nespolo, Makoto Tokuda, Akira Yoshiasa
}

\section{To cite this version:}

Massimo Nespolo, Makoto Tokuda, Akira Yoshiasa. The Tricks of the Chameleon. Unexpected Symmetry of the Diffraction Pattern. Crystal Research and Technology, 2019, 54, pp.1900063. 10.1002/crat.201900063 . hal-02270513

\section{HAL Id: hal-02270513 \\ https://hal.univ-lorraine.fr/hal-02270513}

Submitted on 25 Aug 2019

HAL is a multi-disciplinary open access archive for the deposit and dissemination of scientific research documents, whether they are published or not. The documents may come from teaching and research institutions in France or abroad, or from public or private research centers.
L'archive ouverte pluridisciplinaire HAL, est destinée au dépôt et à la diffusion de documents scientifiques de niveau recherche, publiés ou non, émanant des établissements d'enseignement et de recherche français ou étrangers, des laboratoires publics ou privés. 


\section{The tricks of the chameleon. Unexpected Symmetry of the Diffraction Pattern}

${ }^{1}$ Université de Lorraine, CNRS, CRM2, 54000 Nancy, France. ${ }^{2}$ Institute for Materials Research, Tohoku

University, Sendai 980-8577, Japan ${ }^{3}$ Faculty of Advanced Science and Technology, Kumamoto University,

Kumamoto 860-8555, Japan.

Received 17 April 2019. Revised 9 July 2019. Published online 12 August 2019.

\section{Abstract}

The first step in the structure solution process is the determination of candidate space groups on the basis of the

symmetry of the diffraction pattern, in particular through the analysis of the reflection conditions. Sometimes it may 


\section{Introduction}

Space-group symmetry is conveniently and efficiently expressed via the Hermann-Mauguin symbols, from which both

the nature and the orientation in space of symmetry elements is easily obtained. These symbols, as well as the matrix

lattice. The choice of the conventional basis is in general not unique but requires some additional conventions, like the

labelling of the unique axis, when it exists, or choosing one of several possible settings for the orthorhombic groups.

For example, the conventional basis of a tetragonal crystal has one basis vector along the four-fold (unique) axis and the

two other in the plane perpendicular to it, at 90 degrees from each other: the choice to label $\mathbf{c}$ the basis vector along the

unique axis rather than $\mathbf{a}$ or $\mathbf{b}$ is a matter convention and not a restriction due to symmetry. Similarly, choosing the basis

vectors along the symmetry directions of the space-group type No. 62 leads to six different Hermann-Mauguin symbols.

It has been once decided to assign the Pnma the status of "standard" setting, leaving its five siblings (Pmnb, Pbnm,

Pcmn, Pmcn and Pnam) in darkness, although some occasionally do get in the spotlight. This is the case of Pbnm,

which for historical reasons is the favourite setting in which the structure of the minerals of the olivine group is

commonly reported.

The choice of a non-conventional basis is always possible and can be preferable in some cases, like in the study of 
(a series of examples can be found in [1]). The Hermann-Mauguin symbol depends on the choice of the basis vectors

and reflects the setting of the space group; as a result, the number of possible settings, and therefore of the possible

Hermann-Mauguin symbols, even when restricting ourselves to simple and straightforward changes of basis, is much

Also in the case of unconventional settings unusual symbols of space groups are often obtained, like $B 2_{1} / e$ for $P 2_{1} / c$,

For example, $C 2 / c$ becomes $I 2 / a$ by basis vector transformation $(\mathbf{a}, \mathbf{b}, \mathbf{c})_{[2 / a}=(\mathbf{c}, \mathbf{b},-\mathbf{a}-\mathbf{c})_{C 2 / c}(e . g$. gypsum: [2]). An even

$(\mathbf{a}, \mathbf{b}, \mathbf{c})_{P b n m}=(\mathbf{c}, \mathbf{a}, \mathbf{b})_{P n m a}$, the inverse transformation $(\mathbf{a}, \mathbf{b}, \mathbf{c})_{P n m a}=(\mathbf{b}, \mathbf{c}, \mathbf{a})_{P b n m}$. Quite obviously, if the data collection is

obtained in the alternative setting, the structure cannot be solved in standard setting: either the Laue indices of the

reflections are transformed - i.e. $(h k l)_{P n m a}=(k l h)_{P b n m}$ - or the structure is solved in the alternative setting. 
because by definitions the three directions corresponding to the cubic basis vectors are made equivalent by the three-

\section{Alternative descriptions of crystal structures in different space-group settings}

The symmetry of a crystal structure is described by its space group, i.e. a group of isometries (distance-preserving

The group formed by all the isometries of $\mathrm{E}^{3}$ is known as the Euclidean group $\mathcal{E}(3)$; every space group is a subgroup of 
to $G$, where $i$ is the index of $G$ in $\mathcal{K}$, i.e. the ratio of the orders of $\mathcal{K}$ and $G$ :

$$
i=|\mathcal{K}| /|G|[4]
$$

80

The right coset decomposition is written as follows:

$$
\mathcal{K}=\cup_{\mathrm{j}=1, i} G k_{\mathrm{i}}, k_{\mathrm{l}}=1, \kappa_{\mathrm{i}} \notin G k_{\mathrm{k}}, \forall \mathrm{k}<\mathrm{j}
$$

which simply means that the first coset is the subgroup itself $\left(k_{1}=1\right.$, thus $\left.G k_{b}=G\right)$ and the operation $k_{\mathrm{i}}$ does not occur in

any of the previous cosets $(\mathrm{k}<\mathrm{j})$. The decomposition can also be obtained in terms of left cosets:

$$
\mathcal{K}=\cup_{\mathrm{j}=1, i} k_{\mathrm{i}} G, k_{\mathrm{l}}=1, \kappa_{\mathrm{i}} \notin \notin \kappa_{\mathrm{k}} G, \forall \mathrm{k}<\mathrm{j} .
$$

Depending on whether the two decompositions (2) and (3) are identical or not, the relation between $\mathcal{K}$ and $G$ is different.

If the two decompositions (2) and (3) do not give the same result, then, in general, $\kappa_{i} G k_{i}^{-1}=G^{\prime}$. By taking all the $i$

operations $k_{\mathrm{i}}, \mathrm{j}=[1, i]$, where $i$ is the index in Eq. (1), one gets a set of $n$ subgroups $(2 \leq n \leq i) G, G^{\prime}, G^{\prime \prime} \ldots$ which are

conjugated subgroups of $\mathcal{K}$. Conjugation is a similarity transformation and groups $G, G, G^{\prime \prime} \ldots$ are all of the same type,

i.e. they have the same Hermann-Mauguin symbol when expressed in their conventional basis; at least some of their

symmetry elements, however, are differently oriented or located in space.

If the two decompositions (2) and (3) give the same result, then $k_{i} G=G k_{i}$ for any $k_{i}$. This result can be rewritten as $k_{i} G k_{i}^{-1}$

$=G, \forall \xi_{i} \cdot G$ is a normal (invariant, self-conjugate) subgroup of $\mathcal{K}$ : in symbols, $G \triangleleft \mathcal{K}$.

If $G$ is not a normal subgroup of $\mathcal{K}$, then one can always find an intermediate group $\mathcal{M}$ such that $\mathcal{G}$ is a normal subgroup 
representation of the symmetry operations is unchanged under the action of $\mathcal{N}_{\mathcal{E}}(G)$ or $\mathcal{N}_{\mathcal{A}}(G)$ so that the Hermann-

Mauguin symbol of $G$ is not affected when the crystal structure description is modified by an operation of these

normalizers.

The structure of rock salt, space-group type $F m \overline{3} m$, can be described with $\mathrm{Na}$ in position $4 a(0,0,0)$ and $\mathrm{Cl}$ in position

$4 b(1 / 2,1 / 2,1 / 2)$ or with $\mathrm{Cl}$ in position $4 a$ and $\mathrm{Na}$ in position $4 b$. The normalizer of the space group is $\mathcal{N}_{\mathcal{E}}(F m \overline{3} m)=P m \overline{3} m$

with basis vectors $\mathbf{a} / 2, \mathbf{b} / 2, \mathbf{c} / 2$ with respect to those of $F m \overline{3} m$; the coset decompositions gives two cosets, the group

$F m \overline{3} m$ itself and a second coset $F m \overline{3} m \cdot t(1 / 2,1 / 2,1 / 2)$. Any of the operations in this coset, obviously including the additional

generator $t(1 / 2,1 / 2,1 / 2)$, map the Wyckoff position $4 a$ of $F m \overline{3} m$ onto the Wyckoff position $4 b$ and vice versa. The only

degree of freedom in a cubic space group being the value of the single independent cell parameter, $\mathcal{N}_{\mathcal{A}}(G)=\mathcal{N}_{\mathcal{E}}(G)$ for

cubic crystals. This explains why there are two alternative but equivalent descriptions of rock salt: they are related by

the operations of $\mathcal{N}_{\mathcal{E}}(F m \overline{3} m)$ which are not in $F m \overline{3} m$. Because $F m \overline{3} m$ is normal in $\mathcal{N}_{\mathcal{E}}(F m \overline{3} m)$, the two descriptions of the

structure correspond to the same Hermann-Mauguin symbol.

In case of metric specialisation, the Euclidean normalizer is a supergroup of that corresponding to the general (non-

specialized) metric: it is called an enhanced Euclidean normalizer (the enhanced Euclidean normalizer corresponding to

the highest possible metric specialization coincides with the affine normalizer). As a consequence, the number of

equivalent descriptions of the crystal structure increases. For example, a compound crystallising in a space group of

type Pccn (No. 49) has $\mathcal{N}_{\mathcal{E}}(G)=$ Pmmm with basis vectors $\mathbf{a} / 2, \mathbf{b} / 2, \mathbf{c} / 2$. The additional generators of $\mathcal{N}_{\mathcal{E}}(G)$ with respect 
and the operations in the corresponding cosets). Moreover, $\mathcal{N}_{\mathcal{A}}(G)=P 4 / \mathrm{mmm}$, which corresponds to the enhanced

Euclidean normalizer for $a=b$, with basis vectors $\mathbf{a} / 2, \mathbf{b} / 2, \mathbf{c} / 2$, with additional generator the reflection $m x, x, z$ with

respect to $\mathcal{N}_{\mathcal{E}}(G)$. This means that if $a=b$, then the same structure can also be described in the same axial setting of the

same space group in eight more ways (thus sixteen in total), related to the other eight descriptions by a reflection about

a (110) plane passing through the origin (as well as all the operations in the corresponding cosets). The two other

tetragonal metric specialisation, i.e. $b=c$ or $a=c$, do not lead to enhanced Euclidean normalizers, so that the number of

equivalent descriptions does not increase: in fact, the symmetry elements along the two directions of specialisation are

not of the same type ( $c$ and $n$ respectively). Similarly, a cubic metric specialisation, i.e. $a=b=c$, does not increase the

number of equivalent descriptions with respect to $\mathcal{N}_{\mathcal{A}}(G)$. Now, operations that do not belong to the affine normaliser

result in alternative but non-equivalent descriptions of the crystal structure and also modify the Hermann-Mauguin

symbol of the space group. For example, three-fold rotations about the [111] direction (as well as any operation in the

corresponding coset) change the space-group symbol from Pccm to Pmaa - basis vector change $(\mathbf{a}, \mathbf{b}, \mathbf{c})_{P_{\text {maa }}}=(\mathbf{c}, \mathbf{a}, \mathbf{b})_{P_{\text {Pccm }} \text { - }}$

or $P b m b$ - basis vector change $(\mathbf{a}, \mathbf{b}, \mathbf{c})_{P b m b}=(\mathbf{b}, \mathbf{c}, \mathbf{a})_{P c c m}$.

\section{Data collection in alternative settings of the space-group}

Data collection from diffractometers equipped with point detectors required the user to choose the unit cell on the basis

of a limited number of diffractions, before the data collection actually started. The introduction of area detectors have

made this step no longer necessary: the unit cell can be chosen a posteriori and often in a rather automatic way, 
setting, or an alternative setting of the space group has to be used for the structure solution and refinement. Modern

software packages are usually able to recognize the choice of an alternative setting, and to operate the transformation to

a standard setting. This is however not always the case and the investigator should be aware that the automatic indexing

may sometimes result in an unusual setting. This may induce mistakes in subsequent structure analysis, as we are going

to show with a couple of concrete examples.

\subsection{Overlooked symmetry in analcime}

A textbook example was reported recently on analcime, a mineral belonging to the family of feldspathoids which

crystallizes in a cubic space group of type $I a \overline{3} d$ or in its translationengleiche subgroup of type $I 4_{1} / a c d$. Sugano \& Kyono

[7] investigated two tetragonal synthetic samples that had been reheated for 24 and 48 hours respectively. As reminded

in the Introduction, the choice of labelling $\mathbf{c}$ the basis vector along the unique axis of a tetragonal crystal is a matter of

convention and not imposed by any symmetry consideration. For reasons that are unclear (no CIF file was provided) the

diffraction data [7] were indexed in one case in an $a$-unique setting, and in the other case in a $b$-unique setting. These

unusual settings misled to authors to think their samples were orthorhombic, because no systematic absences from the

fourfold screw axis were observed along the $00 l$ row (they were along the $h 00$ and $0 k 0$ rows respectively) and to draw

conclusions about cation ordering that turned out to be contradictory with respect to the experimental data, once the

latter were re-indexing in the conventional $c$-unique setting [8]. The incorrect interpretation was likely the result of no 
basis vectors in common with those the space group. The operations of $P a \overline{3}$, but also those of $\mathcal{N}_{\mathcal{E}}(\operatorname{Pa} \overline{3})=\mathcal{N}_{\mathcal{A}}(P a \overline{3})=I a \overline{3}$,

leave the Hermann-Mauguin symbol of the space group unchanged. However, any of the operations of a cubic

holohedral supergroup of $I a \overline{3}$ do produce an alternative, unconventional setting. In particular, their effect on the glide

planes is the following:

$a x, y, 1 / 4$ is transformed to $b x, y, 1 / 4$

$c x, 1 / 4, z$ is transformed to $c^{1 / 4, y, z}$

$b^{1 / 4, y, z}$ is transformed to $a x, 1 / 4, z$

The Hermann-Mauguin symbol of the space group in this setting is $P b \overline{3}$. The Wyckoff positions are transformed as in

Table 1 , where as coset representative a twofold rotation about [1 $1 \overline{1} 0]$ has been chosen. If the data collection - the $h k l, I, \sigma$

file - is indexed in $P b \overline{3}$ but the structure refinement is attempted in $P a \overline{3}$, a large number of violations of reflection

conditions occur and the refinement obviously fails. The unaware investigator may be trapped in thinking he is dealing

with a crystal of lower symmetry, possibly twinned, whereas he is simply using an unsuitable setting of the space group.

This is precisely the situation we had to deal with, as described in the following section.

\subsection{Experimental and results}

A sample of natural pyrite, $\mathrm{FeS}_{2}$, in the shape of a pentagonal-dodecahedron, was obtained from Hechi, Guangxi, China.

Data collection was performed on a Rigaku SuperNova single source at offset/far, HyPix3000 diffractometer under the

program CrysAlisPro (Version 1.171.39.27b). The intensity of reflections was measured using graphite-monochromated

MoKa radiation (0.71069 $\AA$ ). CrysAlisPro program was used to refine the cell dimensions and for data reduction. The 
reflections with $\left.F_{\mathrm{O}}>3 \sigma_{(\mathrm{O}}\right)(195$ in number) were used to refine the crystal structure by the full matrix-least-square

method, which was performed using Jana 2006 [9].

The structure of pyrite being well-known, we assumed at first a space group of type $\mathrm{Pa} \overline{3}$. The set of reflections indexed

in $\mathrm{Pa} \overline{3}$ included 65 violations of the reflection conditions. The average $\mathrm{I} / \sigma$ ratio of these reflections was 10.78 , with the

strongest reflections showing a ratio above 80 . These violations cannot therefore be considered outliers. Not

surprisingly, using the coordinates of $\mathrm{Fe}$ and $\mathrm{S}$ in $\mathrm{Pa} \overline{3}$ resulted in very poor quality refinement: $\mathrm{R}(\mathrm{obs})=32.4 \%$, $\mathrm{R}$ (all)

$=44.3 \%, \mathrm{GoF}=22.4$.

The Laue indices of the reflections violating the systematic absences of $P a \overline{3}$ correspond to zonal reflection conditions.

One may therefore be tempted to think one is dealing with a sample whose actual symmetry is $P 2{ }_{1} 3$, possibly twinned,

in which no violation of reflection conditions appear. The refinement in $P 2{ }_{1} 3$ does converge to $\mathrm{R}(\mathrm{obs})=2.60 \% \mathrm{R}($ all $)=$

$0.3652(2)$, S2 $0.8651(2) 0.6349(2) 0.3651(2)$. The results is however very strongly pseudo-symmetric with respect to

$\operatorname{Pa} \overline{3}$, to which it is transformed by a change of basis vectors $(\mathbf{a}, \mathbf{b}, \mathbf{c})_{P b \overline{3}}=(-\mathbf{b},-\mathbf{a},-\mathbf{c})_{P a \overline{3}}$ with origin shift $3 / 4,3 / 4,3 / 4$, as can be

verified with the routine PSEUDO at the Bilbao Crystallographic Server [10],[11]. This permutation of basis vectors

corresponds to the twofold rotation [1 $\overline{1} 0]$ chosen to obtain the Wyckoff positions of $P b \overline{3}$ from those of $P a \overline{3}$ (Table 1),

which shows that the origin of the apparent discrepancy with respect to the structural data already available in the 


\section{Conclusions}

The use of alternative axial space-group settings has definite advantages for several problems [1]. However,

when the investigator has to face experimental data expressed in such a setting he may be led to supposing

his sample is less symmetric than what it actually is. We have discussed two examples: one from the

literature, in which the authors did not realize they were dealing with an alternative setting of the space

group, and one from our own data, which is more subtle, because the unconventional setting $P b \overline{3}$ of the space

group does not appear either in the list of space-group symbols in the International Tables for

Crystallography - it is only mentioned in the sixth edition [12] at the end of section 1.5.4.5.3 and in Table

\section{Acknowledgements}

Critical remarks by two anonymous reviewers are thankfully acknowledged.

\section{Conflict of interest}

The authors declare no conflict of interest. 
[1] M. Nespolo, M.I. Aroyo, Acta Crystallogr. 2016, A72, 523.

[2] P.F. Schofield, K.S. Knight, I.C. Stretton, Am. Mineral. 1996, 81, 847.

[3] To the best of our knowledge, the first to point out the neglected existence of two settings of this type of space

[4] The order of a space group is always infinite, but the index of a subgrou, which is a space group itself is finite.

[5] E. Koch, W. Fischer, Z. Kristallogr. 2006, 221, 1.

[6] M. Nespolo, J. Appl. Crystallogr. 2015, 48, 1985.

[7] N. Sugano, A. Kyono, Phys. Chem. Miner. 2018, 45, 381.

[8] M. Nespolo, Phys. Chem. Miner. 2018, 45, 391.

[9] V. Petříček, M. Dusek, L. Palatinus, Z. Kristallogr. 2014, 229, 345.

[10] C. Capillas, E.S. Tasci, G. de la Flor, D. Orobengoa, J.M. Perez-Mato, M.I. Aroyo, Z. Kristallogr. $2011,226,186$.

[11] M.I. Aroyo, J.M. Perez-Mato, C. Capillas, E. Kroumova, S. Ivantchev, G. Madariaga, A. Kirov, H. Wondratschek,

[12] M.I. Aroyo (Ed.), International Tables for Crystallography Volume A: Space-group symmetry. Wiley, Hoboken,

[14] W.R. Busing, H.A. Levy, Acta Crystallogr. 1957, 10, 180. 
Table 1. Wyckoff positions in $P a \overline{3}$ and in $P b \overline{3}$.

$\mathrm{Pa} \overline{3}$

Posi Site-

Coordinates

tion symmetry

group

$24 d$

$$
\begin{aligned}
& (x, y, z)(\bar{x}+1 / 2, \bar{y}, z+1 / 2) \\
& (\bar{x}, y+1 / 2, \bar{z}+1 / 2)(x+1 / 2, \bar{y}+1 / 2, \bar{z}) \\
& (z, x, y)(z+1 / 2, \bar{x}+1 / 2, \bar{y}) \\
& (\bar{z}+1 / 2, \bar{x}, \mathrm{y}+1 / 2)(\bar{z}, x+1 / 2, \bar{y}+1 / 2) \\
& (y, z, x)(\bar{y}, z+1 / 2, \bar{x}+1 / 2) \\
& (y+1 / 2, \bar{z}+1 / 2, \bar{x})(\bar{y}+1 / 2, \bar{z}, x+1 / 2) \\
& (\bar{x}, \bar{y}, \bar{z})(x+1 / 2, y, \bar{z}+1 / 2) \\
& (x, \bar{y}+1 / 2, z+1 / 2)(\bar{x}+1 / 2, y+1 / 2, z) \\
& (\bar{z}, \bar{x}, \bar{y})(\bar{z}+1 / 2, x+1 / 2, y) \\
& (z+1 / 2, x, \bar{y}+1 / 2)(z, \bar{x}+1 / 2, y+1 / 2) \\
& (\bar{y}, \bar{z}, \bar{x})(y, \bar{z}+1 / 2, x+1 / 2) \\
& (\bar{y}+1 / 2, z+1 / 2, x)(y+1 / 2, z, \bar{x}+1 / 2)
\end{aligned}
$$

$8 c$

.3. $(x, x, x)(\bar{x}+1 / 2, \bar{x}, x+1 / 2)$

$(\bar{x}, x+1 / 2, \bar{x}+1 / 2)(x+1 / 2, \bar{x}+1 / 2, \bar{x})$

$(\bar{x}, \bar{x}, \bar{x})(x+1 / 2, x, \bar{x}+1 / 2)$

$(x, \bar{x}+1 / 2, x+1 / 2)(\bar{x}+1 / 2, x+1 / 2, x)$

$4 b$

. 3 . $\quad(1 / 2,1 / 2,1 / 2)(0,1 / 2,0)(1 / 2,0,0)$

$(0,0,1 / 2)$

$4 a$

.3. $\quad(0,0,0)(1 / 2,0,1 / 2)(0,1 / 2,1 / 2)$

$(1 / 2,1 / 2,0)$
Reflection conditions

( $h, k, l$ cyclically

permutable)

$$
0 k l: k=2 n
$$

$h 00: h=2 n$

$(x, y, z)(\bar{x}, \bar{y}+1 / 2, z+1 / 2)$

$(x+1 / 2, \bar{y}, \bar{z}+1 / 2)(\bar{x}+1 / 2, y+1 / 2, \bar{z})$

$(y, z, x)(\bar{y}+1 / 2, z+1 / 2, \bar{x})$

$(\bar{y}, \bar{z}+1 / 2, x+1 / 2)(y+1 / 2, \bar{z}, \bar{x}+1 / 2)$

$(z, x, y)(z+1 / 2, \bar{x}, \bar{y}+1 / 2)$

$(\bar{z}+1 / 2, x+1 / 2, \bar{y})(\bar{z}, \bar{x}+1 / 2, y+1 / 2)$

$(\bar{x}, \bar{y}, \bar{z})(x, y+1 / 2, \bar{z}+1 / 2)$

$(\bar{x}+1 / 2, y, z+1 / 2)(x+1 / 2, \bar{y}+1 / 2, z)$

$(\bar{y}, \bar{z}, \bar{x})(y+1 / 2, \bar{z}+1 / 2, x)$

$(y, z+1 / 2, \bar{x}+1 / 2)(\bar{y}+1 / 2, z, x+1 / 2)$

$(\bar{z}, \bar{x}, \bar{y})(\bar{z}+1 / 2, x, y+1 / 2)$

$(z+1 / 2, \bar{x}+1 / 2, y)(z, x+1 / 2, \bar{y}+1 / 2)$

no extra conditions

$(x, x, x)(\bar{x}, \bar{x}+1 / 2, x+1 / 2)$

no extra conditions

$(x+1 / 2, \bar{x}, \bar{x}+1 / 2)(\bar{x}+1 / 2, x+1 / 2, \bar{x})$

$(\bar{x}, \bar{x}, \bar{x})(x, x+1 / 2, \bar{x}+1 / 2)$

$(\bar{x}+1 / 2, x, x+1 / 2)(x+1 / 2, \bar{x}+1 / 2, x)$

$h k l: h+k, h+l, k+l=2 n(1 / 2,1 / 2,1 / 2)(1 / 2,0,0)(0,1 / 2,0)$

$(0,0,1 / 2)$

$h k l: h+k, h+l, k+l=2 n(0,0,0)(0,1 / 2,1 / 2)(1 / 2,0,1 / 2)$

$(1 / 2,1 / 2,0)$
Reflection conditions

$(h, k, l$ cyclically permutable)

$0 k l: l=2 n$

$h 00: h=2 n$ 
Temperature (K)

$a(\AA)$

$V\left(\AA^{3}\right)$

Z

Radiation type

$\mu\left(\mathrm{mm}^{-1}\right)$

Crystal size (mm)

Data collection

Diffractometer

Absorption correction

$T \min , T \max$

No. of measured, independent and

observed $[I>3 \sigma(I)]$ reflections

Rint

$(\sin \theta / \lambda) \max \left(\AA^{-1}\right)$

Refinement

$R\left[F^{2}>2 \sigma\left(F^{2}\right)\right], w R\left(F^{2}\right), S$

No. of reflections

No. of parameters

$\Delta \rho \max , \Delta \rho \min \left(\mathrm{e} \AA^{-3}\right)$
$5.4177(1)$

$159.02(1)$

4

Mo $K \alpha$

11.46

$0.06 \times 0.05 \times 0.03$

Rigaku SuperNova

Integration [14]

$0.642,0.786$

1159, 222, 195

0.026

0.994

$0.023,0.028,1.41$

222

6

$0.70-1.45$

Table 3. Atomic coordinates and anisotropic atomic displacement parameters for pyrite in $\mathrm{Pb} \overline{3}$

\begin{tabular}{|c|c|c|c|c|c|c|c|c|c|}
\hline & $x$ & $y$ & $z$ & $U_{11}$ & $U_{22}$ & $U_{33}$ & $U_{23}$ & $U_{13}$ & $U_{12}$ \\
\hline$\overline{\mathrm{Fe}}$ & $1 / 2$ & 0 & 0 & $0.00253(10)$ & $0.00253(10)$ & $0.00253(10)$ & $0.00006(7)$ & $-0.00006(7)$ & $-0.00006(7)$ \\
\hline $\mathrm{S}$ & $0.11511(5)$ & $0.11511(5)$ & $0.11511(5)$ & $0.00302(11)$ & $0.00302(11)$ & $0.00302(11)$ & $-0.00002(8)$ & $-0.00002(8)$ & $-0.00002(8)$ \\
\hline
\end{tabular}

Table 4. Interatomic bond distances $(\AA)$ and angles $\left(^{\circ}\right)$ for pyrite in $P b \overline{3}$

Atom 1 Atom 2 Atom 3

$\begin{array}{lllr}\text { Fe } & \text { S } & --- & 2.2641(3) \times 6 \\ \text { S } & \text { S } & --- & 2.1604(4) \\ \text { S } & \text { Fe } & \text { S } & 94.352(10) \times 3\end{array}$




$\begin{array}{lllr}\mathrm{S} & \mathrm{Fe} & \mathrm{S} & 85.648(10) \times 3 \\ \mathrm{Fe} & \mathrm{S} & \mathrm{Fe} & 115.564(13) \times 3 \\ \mathrm{Fe} & \mathrm{S} & \mathrm{S} & 102.337(14) \times 3\end{array}$

